\title{
An Algorithm for Computing a Nondegenerate Hysteresis Point
}

\author{
By
}

\author{
Hisashi Окамото*
}

\begin{abstract}
We propose an algorithm for computing nondegenerate hysteresis points arising in bifurcation problems with two parameters such as $G: \boldsymbol{R}^{2} \times \boldsymbol{R}^{\mathbb{N}} \rightarrow \boldsymbol{R}^{N}$. A combination of methods in [5] and [7] requires finding zeros of an extended system of $4 N+3$ variables. Shintani and Kanda [6] proposes another algorithm, in which computation of extended system of $3 N+3$ variables is sufficient. On the contrary, in our algorithm, we should find zeros of another extended system of $3 N+2$ variables.
\end{abstract}

\section{§1. Introduction}

In this paper, we consider a problem to find zeros of the equation $G(\lambda, \mu, x)=0$, where $\lambda$ and $\mu$ are real parameters, $x \in \boldsymbol{R}^{N}$ and $G$ is an $N$-vector, i.e., $G: \boldsymbol{R}^{2} \times \boldsymbol{R}^{N} \rightarrow \boldsymbol{R}^{N}$. We assume that $G$ is three times continuously differentiable. We want to compute numerically a hysteresis point, the most elementary example of which is provided by the origin in a one-parameter bifurcation equation

$$
g(\lambda, x)=\lambda-x^{3} \quad(\lambda, x \in \boldsymbol{R}) .
$$

Turning points are generic objects in bifurcation problems with a parameter. The hysteresis point above either splits into two turning points or disappears leaving only ordinary points. The situation is illustrated by a simple example:

$$
G(\lambda, \mu, x)=\lambda-x^{3}-\mu x .
$$

The bifurcation diagrams for $\mu>0, \mu=0$ and $\mu<0$ are shown in Figure 1, 2 and 3 , respectively.

* Partly supported by Ohbayashi Corporation Received December 21, 1990. Revised February 18, 1991.

1991 Mathematics Subject Classification: 65H17

* Research Institute for Mathematical Sciences, Kyoto University, Kyoto, 606-01, Japan. 


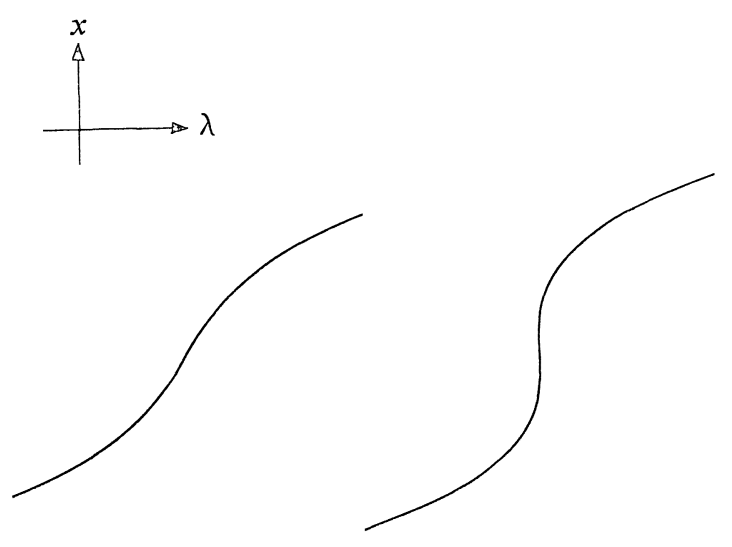

Figure 1

Figure 2

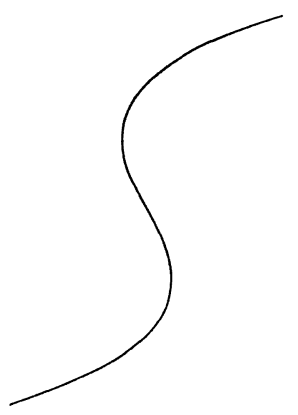

Figure 3

In a vague fashion we call $(\bar{\lambda}, \bar{\mu}, \bar{x})$ a hysteresis point if $G(\bar{\lambda}, \bar{\mu}, \bar{x})=0$ and the bifurcation diagram $D_{\mu} \equiv\{(\lambda, x) ; G(\lambda, \mu, x)=0\}$ has only ordinary points (like Figure 1) for $\mu$ in one side of $\bar{\mu}$ and two turning points (like Figure 3 ) for $\mu$ in another side of $\bar{\mu}$. We give a numerical algorithm for computing a "nondegenerate" hysteresis points. This is possible, since a nondegenerate hysteresis point is structurally stable in two parameter systems. Rigorous definitions are given in $\S 2$. Theorem and its proof are presented in $\S 3$.

\section{§2。 Definition and Algorithm}

We first assume that there exists a $(\bar{\lambda}, \bar{\mu}, \bar{x}) \in \mathbb{R} \times \mathbb{R} \times \mathbb{R}^{N}$ satisfying

$$
G(\bar{\lambda}, \bar{\mu}, \bar{x})=0 \text {. }
$$

Throughout this paper, derivatives are denoted by subscripts and overline indicates that the mapping is evaluated at $(\bar{\lambda}, \bar{\mu}, \bar{x})$. For instance, we write $\bar{G}$ and $\bar{G}_{x}$ instead of $G(\bar{\lambda}, \bar{\mu}, \bar{x})$ and $G_{x}(\bar{\lambda}, \bar{\mu}, \bar{x})$, respectively.

Secondly we assume that the null-space of $\bar{G}_{x}$ is one dimensional and spanned by $\bar{\phi} \in \mathbb{R}^{N} \backslash\{0\}$. Note that the corank of $\bar{G}_{x}$ is also one. Letting $\langle$,$\rangle denote the Euclidian inner product, we assume that$

$$
\text { Range } \bar{G}_{x}=\left\{y \in \mathbb{R}^{N} ;\langle\bar{\psi}, y\rangle=0\right\} \text {. }
$$

We normalize $\bar{\phi}$ and $\bar{\psi}$ by $\langle\bar{\phi}, \bar{\phi}\rangle=1$ and $\langle\bar{\psi}, \bar{\psi}\rangle=1$. We put $g(\lambda, x)=$ $G(\lambda, \bar{\mu}, x)$. We wish to find a point at which the bifurcation diagram of $g(\lambda, x)$ looks like Figure 2. After [7] we make the following

Definaition ([7])。 We call $(\bar{\lambda}, \bar{x})$ a nondegenerate turning point of $g$ if 
$\left\langle\bar{\psi}, \bar{g}_{\lambda}\right\rangle \neq 0$ and $\left\langle\bar{\psi}, \bar{g}_{x x} \bar{\phi} \bar{\phi}\right\rangle \neq 0$, where $\bar{g}_{x x}$ is the bilinear operator representing the second order derivative of $g$ and $\bar{g}_{x x} \bar{\phi} \bar{\phi}$ is the value of $\bar{g}_{x x}$ taken at $(\bar{\phi}, \bar{\phi})$. It is called a nondegenerate hysteresis point if $\left\langle\bar{\psi}, \bar{g}_{\lambda}\right\rangle \neq 0,\left\langle\bar{\psi}, \bar{g}_{x x} \bar{\phi} \bar{\phi}\right\rangle=0$ and the condition (3.3) of the next section holds.

We can understand the meaning of this definition by the example $g(\lambda, x)=$ $\alpha_{0} \lambda+\alpha_{2} x^{2}+\alpha_{3} x^{3}+\cdots$, where $x$ is a scalar. Since we are dealing with bifurcation equations, the term which is linear in $x$ does not appear. The condition $\left\langle\bar{\psi}, \bar{g}_{\lambda}\right\rangle \neq 0$ corresponds to $\alpha_{0} \neq 0$ and $\left\langle\bar{\psi}, \bar{g}_{x x} \bar{\phi} \bar{\phi}\right\rangle \neq 0$ to $\alpha_{2} \neq 0$. Such a bifurcation diagram as $\alpha_{0} \alpha_{2} \neq 0$ produces a diagram containing a turning point (=limit point) and is a generic object in one parameter problems. On the other hand, there appears $g$ with $\alpha_{2}=0$ if $g$ is perturbed by $\mu$. In this case it is natural to adopt the assumption $\alpha_{3} \neq 0$ as a nondegeneracy condition. This corresponds to the condition (3.3) below. Accordingly we assume that

$$
\left\langle\bar{\psi}, \bar{G}_{x x} \bar{\phi} \bar{\phi}\right\rangle=0 \text {. }
$$

Since $\bar{\phi} \neq 0$, there is a $k \in\{1,2, \cdots, N\}$ such that the $k$-th component $\phi_{k} \neq 0$. We fix this $k$ hereafter. We define a projection $P$ by

$$
P\left(x_{1}, x_{2}, \cdots, x_{N}\right)=\left(x_{1}, \cdots, x_{k-1}, 0, x_{k+1}, \cdots, x_{N}\right) .
$$

In an obvious way, we identify the range of $P$ with $\boldsymbol{R}^{N-1}$. We now consider the following mapping:

$$
\begin{gathered}
F: \mathbb{R}^{2} \times \mathbb{R}^{N} \times \mathbb{R}^{N} \times \mathbb{R}^{N} \rightarrow \mathbb{R}^{3} \times \mathbb{R}^{N} \times \mathbb{R}^{N} \times \mathbb{R}^{N-1} \\
F(\lambda, \mu, x, \phi, \psi)=(\langle\phi, \phi\rangle-1,\langle\psi, \psi)\rangle-1,\left\langle\psi, G_{x x}(\lambda, \mu, x) \phi \phi\right\rangle, \\
\left.G(\lambda, \mu, x), G_{x}(\lambda, \mu, x) \phi, P G_{x}(\lambda, \mu, x)^{t} \psi\right),
\end{gathered}
$$

where $\lambda, \mu \in \mathbb{R}, x, \phi, \psi \in \boldsymbol{R}^{N}$ and the superscript $t$ implies the transposed matrix. We see that $\bar{F} \equiv F(\bar{\lambda}, \bar{\mu}, \bar{x}, \bar{\phi}, \bar{\psi})=0$. Thus we should compute zeros of $F$. The point of the definition of $F$ is the use of $P$. If we use $G_{x}(\lambda, \mu, x)^{t} \psi$ instead of $P G_{x}(\lambda, \mu, x)^{t} \psi$ in the definition of $F$, then $F$ is a mapping from $\mathbb{R}^{2} \times \mathbb{R}^{3 N}$ into $\mathbb{R}^{3} \times \mathbb{R}^{3 N}$ and becomes formally overdetermined. Since we need to have $\psi$ such that $G_{x}(\lambda, \mu, x)^{t} \psi=0$ (see (2.1)), the reader might think that information about $\psi$ is lost if we adopt (2.3) as the definition. The following lemma guarantees that we do not lose the information.

Lemma. If $F(\lambda, \mu, x, \phi, \psi)=0$, then $G_{x}(\lambda, \mu, x)^{t} \psi=0$.

Proof. We put $y=G_{x}(\lambda, \mu, x)^{t} \psi$. By the assumption, all but the $k$-th component of $y$ vanish. By $0=\left\langle G_{x}(\lambda, \mu, x) \phi, \psi\right\rangle=\langle\phi, y\rangle=\phi_{k} y_{k}$ and $\phi_{k} \neq 0$, 
we obtain $y_{k}=0$.

This lemma shows that finding zeros of $F$ is equivalent to finding $(\lambda, \mu, x, \phi, \psi)$ such that $\langle\phi, \phi\rangle=1,\langle\psi, \psi\rangle=1, G(\lambda, \mu, x)=0, G_{x}(\lambda, \mu, x) \phi=0$, $G_{x}(\lambda, \mu, x)^{t} \psi=0$ and $\left\langle\psi, G_{x x}(\lambda, \mu, x) \phi \phi\right\rangle=0$.

The algorithm which we propose in this paper is to apply a Newton method to $F$. We are now in a position to compare the algorithms in $[1,5,7]$ with ours. In order to compute turning points of $G,[1,5]$ uses the following extended system:

$$
H(\mu, y)=\left(l \phi-1, G(\lambda, \mu, x), G_{x}(\lambda, \mu, x) \phi\right),
$$

where $y=(\lambda, x, \phi)$ and $l$ is an appropriate linear functional on $\mathbb{R}^{N}$. Let $Y=\mathbb{R} \times \mathbb{R}^{N} \times \mathbb{R}^{N}$. Then $H$ is a mapping from $\mathbb{R} \times Y$ into $Y$. Note that this extended system $H$ has $2 N+1$ variables, while $G$ has $N$ variables. They proved in $[1,5]$ under a reasonable assumption that a nondegenerate hysteresis point $(\bar{\lambda}, \bar{\mu}, \bar{x})$ with respect to $\lambda$ corresponds to a nondegenerate turning point $(\bar{\mu}, \bar{y})$ of $H$ with respect to $\mu$. Therefore, if we trace the curve of $H=0$, e.g, by Keller's method ([4]), and if we find a turning point of $H$, then we will obtain a nondegenerate hysteresis point of $G$. In order to get a precise location of the hysteresis point (i.e., the turning point of $H=0$ ), we should utilize the technique in [5]. Their algorithm is as follows: after getting a good approximation to the turning point to $H=0$ we consider an extended system of $H$. Namely,

$$
T(\mu, y, \Phi)=\left(m \Phi-1, H(\mu, y), H_{y}(\mu, y) \Phi\right) \quad(\mu \in \mathbb{R}, y \in Y, \Phi \in Y) .
$$

They proved in [5] that a nondegenerate turning point of $H$ is an ordinary point of $T$. Using the approximation as an initial value to a Newton iteration to $T$, we get a very accurate approximation to the turning point of $H$. Consequently, we have to solve $T$, which, as an extended system of $H$, contains $2 \cdot(2 N+1)+1=4 N+3$ variables. On the other hand, our algorithm is more direct and contains only $3 N+2$ variables. Therefore we propose to perform the computation as follows:

First, trace the curve of $H=0$ and get approximate locations of hysteresis points (=turning points of $H$ ). If we need more precise information, then solve $F=0$ by the Newton method.

Remark 1. In the case where $N=1$, the last term of the right hand side of (2.3) disappears and no difficulty arises (see [2]). The use of the projection $P$, which depends on $\bar{\phi}$, may cause a problem in some cases. We thereby propose to use our algorithm when we already have an approximation $\bar{\phi}^{*}$ to 
$\bar{\phi}$. In this case, the index $k$ arising in the definition of $P$ can be taken as the one where the maximum of the absolute values of the components is attained. Thus, if our aim is to get a very accurate approximation from a rough approximation, the use of $\mathbb{P}$ seems to cause little difficulty.

Finally we compare our method with the one in [6]. They consider the following map: $S: \mathbb{R}^{2} \times \mathbb{R}^{2 N} \times \mathbb{R} \times \mathbb{R}^{N} \rightarrow \mathbb{R}^{2 N} \times \mathbb{R} \times \mathbb{R}^{N} \times \mathbb{R}^{2}$;

$$
S(\mu, \lambda, x, \phi, u)=(E(\mu, \lambda, \phi), D(\mu, y, \phi, u),\langle(0, \phi), u\rangle,\langle(1,0), u\rangle),
$$

where $y=(\lambda, x), u \in \mathbb{R} \times \mathbb{R}^{N}$,

$$
E(\mu, \lambda, \phi)=\left(G(\lambda, \mu, x), G_{x} \phi,\langle\phi, \phi\rangle-1\right)
$$

and $D(\mu, y, \phi, u)=G_{x x} \phi \phi+G_{(\lambda, x)} u$. The number of variables in the equation is $3 N+3$, which is almost the same as that of ours. Furthermore, their method can be applied to the infinite dimensional case while our method is applicable only to the finite dimensional case. We do not know which method is better in actual computations. We leave the comparison in the future works.

\section{§. Theorem and Proof}

Our aim in this paper is to show that the derivative $D \bar{F}$ is nonsingular under a certain assumption. We define several quantities. First,

$$
\bar{d}=\left\langle\bar{\psi}, \bar{G}_{\lambda}\right\rangle\left\langle\bar{\psi}, \bar{G}_{\mu_{x}} \bar{\phi}\right\rangle-\left\langle\bar{\psi}, \bar{G}_{\mu}\right\rangle\left\langle\bar{\psi}, \bar{G}_{\lambda x} \bar{\phi}\right\rangle .
$$

$\bar{v}$ is defined at a solution to

$$
\bar{G}_{x} \bar{v}=-\left\langle\bar{\psi}, \bar{G}_{\mu}\right\rangle \bar{G}_{\lambda}+\left\langle\bar{\psi}, \bar{G}_{\lambda}\right\rangle \bar{G}_{\mu},\langle\bar{\phi}, \bar{v}\rangle=0 .
$$

$\bar{u}$ is defined as a solution to

$$
\bar{G}_{x} \bar{u}=-\bar{G}_{x x} \bar{\phi} \bar{\phi},\langle\bar{\phi}, \bar{u}\rangle=0 .
$$

The vector $\bar{u}$ is well-defined by (2.2).

We assume that the following (3.1-3) hold true:

$$
\begin{gathered}
\bar{d} \neq 0, \\
\left\langle\bar{\psi}, \bar{G}_{x x} \bar{\phi} \bar{v}\right\rangle \neq \bar{d}, \\
\left\langle\bar{\psi}, \bar{G}_{x x x} \bar{\phi} \bar{\phi} \bar{\phi}\right\rangle+3\left\langle\bar{\psi}, \bar{G}_{x x} \bar{\phi} \bar{u}\right\rangle \neq 0 .
\end{gathered}
$$

Theorem. Suppose $(3.1-3)$ holds true. Then the $(3 N+2) \times(3 N+2)$ matrix $D \bar{F}$ is nonsingular. 
Proof. We show the injectivity. Suppose that $D \bar{F}(\delta \lambda, \delta \mu, \delta x, \delta \phi, \delta \psi)=0$. We observe that $D \bar{F}$ is represented as follows:

$$
\left(\begin{array}{ccccc}
0 & 0 & 0 & 2\langle\bar{\phi}, \circ\rangle & 0 \\
0 & 0 & 0 & 0 & 2\langle\bar{\psi}, \circ\rangle \\
a_{\lambda} & a_{\mu} & \left\langle\bar{\psi}, \bar{G}_{x x x} \bar{\phi} \bar{\phi} \circ\right\rangle & 2\left\langle\bar{\psi}, \bar{G}_{x x} \bar{\phi} \bar{\circ}^{\circ}\right\rangle & \left\langle\circ, \bar{G}_{x x} \bar{\phi} \bar{\phi}\right\rangle \\
\bar{G}_{\lambda} & \bar{G}_{\mu} & \bar{G}_{x} & 0 & 0 \\
\bar{G}_{\lambda x} \bar{\phi} & \bar{G}_{\mu x} \bar{\phi} & \bar{G}_{x x} \bar{\phi} & \bar{G}_{x} & 0 \\
P \bar{G}_{\lambda x}^{g} \bar{\psi} & P \bar{G}_{\mu x}^{u} \bar{\psi} & L & 0 & P \bar{G}_{x}^{b}
\end{array}\right)
$$

where $a_{\lambda}=\left\langle\bar{\psi}, \bar{G}_{\lambda x x} \bar{\phi} \bar{\phi}\right\rangle, a_{\mu}=\left\langle\bar{\psi}, \bar{G}_{\mu_{x x}} \bar{\phi} \bar{\phi}\right\rangle, L$ is a linear map from $\mathbb{R}^{N}$ to $\mathbb{R}^{N-1}$ defined as

$$
\langle L u, w\rangle=\left\langle\bar{\psi}, \bar{G}_{x x} u P w\right\rangle \quad\left(w \in \mathbb{R}^{N}\right)
$$

Accordingly, we have

$$
\begin{gathered}
\langle\bar{\phi}, \delta \phi\rangle=0,\langle\bar{\psi}, \delta \psi\rangle=0, \\
\delta \lambda a_{\lambda}+\delta \mu a_{\mu}+\left\langle\bar{\psi}, \bar{G}_{x x x} \bar{\phi} \bar{\phi} \delta x\right\rangle+2\left\langle\bar{\psi}, \bar{G}_{x x} \bar{\phi} \delta \phi\right\rangle+\left\langle\delta \psi, \bar{G}_{x x} \bar{\phi} \bar{\phi}\right\rangle=0, \\
\delta \lambda \bar{G}_{\lambda}+\delta \mu \bar{G}_{\mu}+\bar{G}_{x} \delta x=0, \\
\delta \lambda \bar{G}_{\lambda x} \bar{\phi}+\delta \mu \bar{G}_{\mu_{x}} \bar{\phi}+\bar{G}_{x x} \bar{\phi} \delta x+\bar{G}_{x} \delta \phi=0, \\
\delta \lambda P \bar{G}_{\lambda x}^{s} \bar{\psi}+\delta \mu P \bar{G}_{\mu x}^{s} \bar{\psi}+L \delta x+P \bar{G}_{x}^{t} \delta \psi=0,
\end{gathered}
$$

Taking inner product of $(3.6,7)$ and $\bar{\psi}$, we have

$$
\left[\begin{array}{cc}
\left\langle\bar{\psi}, \bar{G}_{\lambda}\right\rangle & \left\langle\bar{\psi}, \bar{G}_{\mu}\right\rangle \\
\left\langle\bar{\psi}, \bar{G}_{\lambda x}, \bar{\phi}\right\rangle & \left\langle\bar{\psi}, \bar{G}_{\mu_{x}} \bar{\phi}\right\rangle
\end{array}\right]\left[\begin{array}{l}
\delta \lambda \\
\delta \mu
\end{array}\right]=\left[\begin{array}{c}
0 \\
-\left\langle\bar{\psi}, \bar{G}_{x x}, \bar{\phi} \delta x\right\rangle
\end{array}\right] .
$$

We put $p=\left\langle\bar{\psi}, \bar{G}_{x x} \bar{\phi} \delta x\right\rangle$. Then, $\delta \lambda=p\left\langle\bar{\psi}, \bar{G}_{\mu}\right\rangle\left|\bar{d}, \delta \mu=-p\left\langle\bar{\psi}, \bar{G}_{\lambda}\right\rangle\right| \bar{d}$, since we assume (3.1). Now $\delta x$ is characterized by

$$
\bar{G}_{x} \delta x=(p / \bar{d})\left(-\left\langle\psi, \bar{G}_{\mu}\right\rangle \bar{G}_{\lambda}+\left\langle\bar{\psi}, \bar{G}_{\lambda}\right\rangle \bar{G}_{\mu}\right) 。
$$

Consequently, there is a constant $\alpha$ such that $\delta x=(p / \bar{d}) \bar{v}+\alpha \bar{\phi}$. This and (2.2) yields

$$
p=\left\langle\bar{\psi}, \bar{G}_{x x} \bar{\phi}((p / \bar{d}) \bar{v}+\alpha \bar{\phi})\right\rangle=(p / \bar{d})\left\langle\bar{\psi}, \bar{G}_{x x} \bar{\phi} \bar{v}\right\rangle .
$$

By the assumption (3.2), we have $p=0$, hence $\delta \lambda=\delta \mu=0$. Also, $\delta x=\alpha \bar{\phi}$. By (3.4) and (3.7), $\delta \phi$ satisfies $\alpha \bar{G}_{x x} \bar{\phi} \bar{\phi}+\bar{G}_{x} \delta \phi=0$ and $\langle\bar{\phi}, \delta \phi\rangle=0$. Therefore we have $\delta \phi=\alpha \bar{u}_{0}(3.8)$ is now written as

$$
\alpha\left\langle\bar{\psi}, \bar{G}_{x x} \bar{\phi} P w\right\rangle+\left\langle\delta \psi, \bar{G}_{x} P w\right\rangle=0 \quad\left(w \in \mathbb{R}^{N}\right) .
$$


On the other hand, $\alpha\left\langle\bar{\psi}, \bar{G}_{x x} \bar{\phi} \bar{\phi}\right\rangle+\left\langle\delta \psi, \bar{G}_{x} \bar{\phi}\right\rangle=0$ obviously holds. Therefore,

$$
\alpha\left\langle\bar{\psi}, \bar{G}_{x x} \bar{\phi} w\right\rangle+\left\langle\delta \psi, \bar{G}_{x} w\right\rangle=0 \quad\left(w \in \mathbb{R}^{N}\right) .
$$

It is easy to see that there is a unique $\psi_{1}$ such that

$$
\left\langle\bar{\psi}, \bar{G}_{x x} \bar{\phi} w\right\rangle+\left\langle\psi_{1}, \bar{G}_{x} w\right\rangle=0 \quad\left(w \in \mathbb{R}^{N}\right) \text {, and }\left\langle\bar{\psi}, \psi_{1}\right\rangle=0 .
$$

We now have $\delta \psi=\alpha \psi_{1}$ by (3.4). We then consider (3.5), which is written as

$$
\alpha\left\{\left\langle\bar{\psi}, \bar{G}_{x x x} \bar{\phi} \bar{\phi} \bar{\phi}\right\rangle+2\left\langle\bar{\psi}, \bar{G}_{x x} \bar{\phi} \bar{u}\right\rangle+\left\langle\psi_{1}, \bar{G}_{x x} \bar{\phi} \bar{\phi}\right\rangle\right\}=0
$$

By the definition of $\bar{u}$ and $\psi_{1}$, it holds that

$$
\left\langle\psi_{1}, \bar{G}_{x x} \bar{\phi} \bar{\phi}\right\rangle=-\left\langle\psi_{1}, \bar{G}_{x} \bar{u}\right\rangle=\left\langle\bar{\psi}, \bar{G}_{x x} \bar{\phi} \bar{u}\right\rangle
$$

By the assumption (3.3) we obtain $\alpha=0$. Thus we have proved the injectivity. 圈

Remark 2. The condition (3.3) implies that the order of contact is exactly three and is assumed in [7]. The condition (3.2) is, however, different from their (3.5) in [7]. Ours does not include theirs and theirs does not include ours.

Remark 3. Progressing is a numerical experiment in a problem of Kolmogorov flows ([3]). In this problem we are concerned with stationary solutions to the Navier-Stokes equations defined in two dimensional flat tori with different aspect ratios. The Reynolds number and the aspect ratio parameter correspond to $\lambda$ and $\mu$ in the present paper. The results will be reported in the future.

\section{References}

[1] Jepson, A. and Spence, A., Folds in solutions of two parameter systems and their calculation. Part I, SIAM J. Numer. Anal., 22 (1985), 347-368.

[2] The numerical solution of nonlinear equations having several parameters I: scalar equations, SIAM J. Numer. Anal., 22 (1985), 736-759.

[3] Katsurada, M., H. Okamoto, H. and Shōji, M.. Bifurcation of the stationary NavierStokes flows on a 2-D flat torus, to appear in Proc. Katata Workshop on Nonlinear PDE and Applications.

[4] Keller, H.B., Numerical solution of bifurcation and nonlinear eigenvalue problems, in Applications of Bifurcation Theory, P.H. Rabinowitz, ed.. Academic Press (1977), 359384.

[5] Moore, G. and Spence, A., The calculation of turning points of nonlinear equations, SIAM J. Numer. Anal., 17 (1980), 567-576., 
[6] Shintani, H. and Kanda, M. Extended systems for numerical computation of double and triple turning points, Bull. Fac. School Edu., Hiroshima Univ. Part II, 12 (1989), 37-45.

[7] Spence, A. and Werner, B., Non-simple turning points and cusps, IMA J. Numer. Anal., 2 (1982), 413-427. 